\title{
RELATIVISTIC BINARY PULSARS WITH BLACK HOLE COMPANIONS
}

\author{
Eric Pfahl, ${ }^{1}$ Philipp Podsiadlowski, ${ }^{2}$ and Saul Rappaport ${ }^{3}$ \\ Received 2005 February 5; accepted 2005 March 23
}

\begin{abstract}
Binaries containing a stellar mass black hole and a recycled radio pulsar have so far eluded detection. We present a focused investigation of the formation and evolution of these systems in the Galactic disk, highlighting the factors that limit their numbers and the reasons why they may be extremely rare. We surmise that the birthrate of black hole/ recycled pulsar binaries in the Galactic disk is probably no higher than $\sim 10^{-7} \mathrm{yr}^{-1}$ and may be much less, including zero. Simple arguments regarding common-envelope evolution suggest that these binaries should have orbital periods $<10 \mathrm{hr}$ and an average lifetime of $\lesssim 10^{8} \mathrm{yr}$ before coalescence due to the emission of gravitational radiation. We expect that fewer than $\sim 10$ of these compact, relativistic binaries currently reside in the Galactic disk, less than $0.1 \%-1 \%$ of the number of double neutron stars. The discovery of two or more black hole/recycled pulsar binaries using current radio telescopes would tightly constrain certain ideas regarding the evolution of massive stars, dynamical mass transfer, and black hole formation.
\end{abstract}

Subject headings: binaries: close — black hole physics — gravitational waves — pulsars: general — stars: neutron

\section{INTRODUCTION}

Thirty years have passed since the discovery of PSR B1913+ 16 (Hulse \& Taylor 1975), which has the dual distinction of being the first known binary radio pulsar and double neutron star(DNS). The intervening years - particularly the last decadehave seen enormous growth in the observed number and variety of binary radio pulsars. Among the $\sim 100$ known systems (see Stairs 2004 for a representative listing and references), orbital periods range from 90 minutes to several years, most have white dwarf companions with masses from substellar to near the Chandrasekhar limit, three have a stellar companion, and eight probably contain a second neutron star (Stairs 2004; Faulkner et al. 2005).

With a handful of exceptions, most radio pulsars in binary systems are "recycled," characterized by low inferred surface magnetic field strengths of $\lesssim 10^{10} \mathrm{G}$ and spin periods of 2$100 \mathrm{~ms}$. The term "recycled" refers to the accretion of matter and angular momentum by the neutron star, whereby the spin period is reduced to small values (Smarr \& Blandford 1976; Alpar et al. 1982; Joss \& Rappaport 1983). In contrast, the majority of isolated pulsars (hereafter called "normal") have magnetic fields of $10^{11}-10^{13} \mathrm{G}$ and spin periods of $0.1-1 \mathrm{~s}$. Recycled pulsars show much greater stability as clocks, allowing for very precise dynamical measurements if the pulsar is in a binary. Precision timing of a recycled binary pulsar quickly reveals its orbital motion, and if the orbit is sufficiently compact or inclined, relativistic gravity may cause significant deviations from a Keplerian model, as in the classic example of PSR B1913+16.

The eight observed DNSs have a special status among binary pulsars. As exotic endpoints of massive binary stellar evolution, they probe important details of stellar physics. Moreover, since

\footnotetext{
${ }^{1}$ Chandra Fellow; Department of Astronomy, University of Virginia, 530 McCormick Road, Charlottesville, VA 22903; epfahl@virginia.edu.

2 Department of Astrophysics, University of Oxford, Oxford, OX1 3RH, UK; podsi@astro.ox.ac.uk.

3 Department of Physics, Massachusetts Institute of Technology, 77 Massachusetts Avenue, Cambridge, MA 02139; sar@mit.edu.
}

the bright pulsar is recycled, and both components are essentially point masses, a subset of these systems have proved to be extremely rich laboratories for studying post-Newtonian gravity. The newly discovered "double pulsar" binary PSR J07373039 stands out as having a nearly edge-on orbit and an orbital period of $2.4 \mathrm{hr}$, the shortest among the DNSs (Burgay et al. 2003; Lyne et al. 2004). Finally, the known DNSs, especially PSR J0737-3039, imply a large number of similar systems in the Milky Way and distant galaxies that will produce in the next $\sim 1$ Gyr a powerful burst of electromagnetic and gravitational radiation in the final throes of coalescence (e.g., Kalogera et al. 2004).

All the factors that make DNSs exceptional are greatly enhanced if the pulsar's companion is replaced with a canonical $10 M_{\odot}$ black hole. To date, there has been no detection of a binary containing a stellar mass black hole and a recycled pulsar (BHRP). Several earlier theoretical studies provide insight into the formation of BHRPs in the Galactic disk. Narayan et al. (1991) first mentioned these systems, identified a likely formation scenario (see $\S 2$ ), and suggested a birthrate of $\lesssim 10^{-6} \mathrm{yr}$ based on their nondetection. Binary population synthesis studies by Lipunov et al. (1994), Sipior \& Sigurdsson (2002), Voss \& Tauris (2003), and Sipior et al. (2004) theoretically constrain the BHRP birthrate and the expected orbital parameters. Each of these papers also addresses the formation of systems where the radio pulsar is not recycled (see also Belczynski et al. 2002), which are not considered here. These latter systems are certainly interesting and probably much more numerous than BHRPs, although they are not nearly as desirable as astrophysical laboratories. We also do not investigate the formation of BHRPs in globular clusters (Sigurdsson 2003).

Our main goals are to (1) dissect the most important steps and uncertainties in BHRP formation ( $\S \S 2$ and 3), (2) constrain the BHRP birthrate in the Galactic disk ( $\S 4),(3)$ indicate the range of binary parameters for newly formed systems and the currentepoch population ( $(5)$, and (4) evaluate the likelihood of discovering BHRPs in pulsar surveys $(\S 6)$. Each of these topics has received some attention in one or more of the above papers; however, no clear picture has yet emerged, and previous work 
has not examined all the major physical uncertainties. We utilize simple, semianalytic calculations to present a complete portrait of BHRP formation. The next section gives a preview of the major evolutionary hurdles in BHRP formation and is followed by a more detailed and quantitative discussion in $\S 3$.

\section{OVERVIEW OF FORMATION AND UNCERTAINTIES}

In this paper we consider the most obvious formation channel for BHRPs in the Galactic disk, which is qualitatively identical to the standard model for forming the known DNSs (e.g., Bhattacharya \& van den Heuvel 1991; Tauris \& van den Heuvel 2003). ${ }^{4}$ The scenario begins with a massive primordial binary, where the primary star is the neutron star progenitor of mass $\simeq 8$ to $\gtrsim 25 M_{\odot}$. During a phase of dynamically stable mass transfer from the primary, the initially less massive secondary reaches a mass of $\gtrsim 25 M_{\odot}$ and will ultimately leave a black hole remnant. Mass transfer ends when the core of the primary is exposed. The core then explodes as a Type $\mathrm{Ib} / \mathrm{c}$ supernova, leaving behind a neutron star that may have received a large impulsive kick. Before the secondary evolves significantly, the neutron star may be a radio pulsar or an X-ray source accreting from the wind of its massive companion. Because of the extreme binary mass ratio, the second episode of Roche lobe overflow is dynamically unstable. The neutron star is engulfed by the stellar envelope and dragged toward the secondary's core. Following the successful expulsion of the common envelope, the neutron star orbits the core, which then collapses to a black hole; for less massive secondaries, a second neutron star is formed at this stage. Either before, during, or after the spiral-in, the neutron star accretes a small amount of mass and is recycled. We now address several fundamental uncertainties in the evolution of the massive secondary, spiral-in phase, recycling process, and formation of the black hole.

\subsection{Stellar Evolution Uncertainties}

Surprisingly, the evolution of massive stars is still quite poorly understood. It is generally estimated that the minimum mass of a single star that ends its life as a black hole is $\simeq 25 M_{\odot}$ (see Podsiadlowski et al. 2003, hereafter PRH03, for discussion and references). However, Brown et al. (2001) show that when a star less massive than $40-50 M_{\odot}$ loses its hydrogen-rich envelope before helium core burning (case B mass transfer), its core evolution is changed dramatically, and the star will most likely leave a neutron star remnant. Therefore, mass transfer from the secondary in the above BHRP formation scenario must begin after it has completed most or all of its helium core burning phase (case $\mathrm{C}$ mass transfer; see also Wellstein \& Langer 1999). The formation of BHRPs is then very sensitive to the radial evolution of the massive secondary (see also Portegies Zwart et al. 1997) and uncertainties in the stellar physics.

The range of binary separations for case $\mathrm{C}$ mass transfer depends mainly on the difference between the maximum radius of a single star and the radius at the tip of the first giant branch. For massive stars, this range may be as small as a few percent of the radius in some evolutionary models or a factor of $\gtrsim 2$ in others. The result depends on when stars undergo helium core burning (see, e.g., the difference between the evolutionary tracks

\footnotetext{
4 The only alternative scenario for DNS formation, involving the spiral-in of two stellar cores inside a common envelope (Brown 1995), is probably not a viable channel for BHRP formation, since the pulsar would be the second-born compact object and hence not be recycled (see also J. Dewi, Ph. Podsiadlowski, \& A. Sena 2005, in preparation).
}

in Figures 1 and 2 of Pfahl et al. [2002a, hereafter PRP02]). If helium burning occurs only while the star is a blue supergiant, the range of case $\mathrm{C}$ mass transfer is greatly increased compared to when the star burns helium as a red supergiant (by perhaps a factor of 4 or more; J. Dewi et al. 2005, in preparation). The observed distribution of supergiants in the Hertzsprung-Russell diagram (e.g., Humphreys 1984) suggests that stars as massive as $\sim 40 M_{\odot}$ spend a significant fraction of their helium burning phase as both blue and red supergiants. None of the present theoretical models is yet fully consistent with the observations (e.g., Langer \& Maeder 1995).

\subsection{Severity and Implications of the Spiral-in}

For a neutron star sinking into the envelope of a $\gtrsim 25 M_{\odot}$ star, the orbit may have to shrink by a factor of $\gtrsim 10^{3}$ before the envelope can be expelled, according to recent calculations of envelope binding energies (Dewi \& Tauris 2001; PRH03). In order to avoid a merger of the neutron star and stellar core of radius $\sim 1 R_{\odot}$, the initial orbital separation must be $\gtrsim 2000 R_{\odot}$. Stars of mass $25-50 M_{\odot}$ attain maximum radii of $\simeq 1500-$ $2500 R_{\odot}$ (e.g., Hurley et al. 2000), and so the range of orbital separations for successful systems is restricted to $\simeq 2000$ $4000 R_{\odot}$. A star that fills its Roche lobe in such an orbit is very evolved and in most cases has passed through the phase of helium core burning. Thus, in the Brown et al. (2001) picture, we are safe in using $\simeq 25 M_{\odot}$ as the mass threshold for black hole formation.

If the orbits contract by a factor of 1000 , the final separations are $\lesssim 4 R_{\odot}$. However, if the contraction factors are generally $\gtrsim 2000$, which is quite plausible, then it may be that no BHRPs are formed. Another serious complication arises because the secondaries of interest are very evolved prior to the spiral-in. Prodigious stellar winds during the late phases of evolution may generally cause the secondary's Roche lobe to expand faster than the star, precluding mass transfer altogether (see $\S 3.4$; Portegies Zwart et al. 1997; Kalogera \& Webbink 1998; PRH03). On the other hand, if the star can catch its Roche lobe after losing a significant amount of mass, the orbit is wider at the start of the spiral-in and the envelope mass that must be ejected is reduced, giving the system a better chance to survive.

We assume that the neutron star does not accrete at hypercritical rates and collapses to a black hole during the spiral-in (e.g., Chevalier 1993, 1996). If this were to occur generally, as may, in fact, be the case, then neither DNSs nor BHRPs would form by the channel being discussed (e.g., Brown 1995).

\subsection{Recycling}

The neutron star in a BHRP is mildly recycled after accreting a small amount of matter $\left(\lesssim 0.001 M_{\odot}\right)$ from, for example, the wind of its companion prior to the spiral-in, the stellar envelope during the spiral-in itself, or a residual accretion disk after the orbital contraction has halted. For most DNSs, recycling may result during a short phase of stable mass transfer from the Roche lobe-filling core of the secondary if it is sufficiently low in mass and if it is exposed before central helium ignition (Dewi \& Pols 2003; Ivanova et al. 2003). However, this process does not apply to BHRPs, because a secondary's core is too massive for the transfer to be stable.

An interesting possibility is if recycling in BHRPs occurs mainly after the spiral-in, as the neutron star accretes a fraction of the strong wind from the secondary's core. Since the core has most likely passed through the phase of central helium burning, only $\lesssim 10^{4}$ yr remains before it collapses. At the Eddingtonlimited accretion rate of $\simeq 4 \times 10^{-8} M_{\odot} \mathrm{yr}^{-1}$ (for pure helium), 
TABLE 1

Results for Variations in the Standard Model

\begin{tabular}{|c|c|c|c|c|c|c|}
\hline $\begin{array}{l}\text { Model } \\
\text { (1) }\end{array}$ & $\begin{array}{l}\text { Description } \\
\text { (2) }\end{array}$ & $\begin{array}{c}\text { Birthrate } \\
\left(10^{-7} \mathrm{yr}^{-1}\right) \\
(3)\end{array}$ & $\begin{array}{l}\text { Number in } \\
\text { Galaxy } \\
\text { (4) }\end{array}$ & $\begin{array}{c}\left\langle\tau_{m}\right\rangle \\
(\mathrm{Myr}) \\
(5)\end{array}$ & $\begin{array}{l}\langle e\rangle \\
(6)\end{array}$ & $\begin{array}{c}\left\langle P_{b}\right\rangle \\
(\mathrm{hr}) \\
(7)\end{array}$ \\
\hline 1 ………………… & Standard $(\gamma=0.1)$ & 0.63 & 5.4 & 85 & 0.078 & 3.5 \\
\hline $2^{\mathrm{a}} \ldots \ldots \ldots \ldots \ldots \ldots$ & $\gamma=0.07$ & 0.03 & 0.2 & 57 & 0.080 & 3.2 \\
\hline $3^{\mathrm{a}} \ldots \ldots \ldots \ldots \ldots \ldots \ldots \ldots \ldots \ldots \ldots \ldots \ldots$ & $\gamma=0.15$ & 8.80 & 103.0 & 118 & 0.078 & 3.8 \\
\hline $4^{\mathrm{a}} \ldots \ldots \ldots \ldots \ldots \ldots \ldots \ldots \ldots \ldots \ldots \ldots \ldots \ldots$ & $\gamma=0.30$ & 110.00 & 3800.0 & 345 & 0.079 & 5.2 \\
\hline 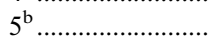 & $p\left(M_{p}^{\mathrm{PB}}\right) \propto\left(M_{p}^{\mathrm{PB}}\right)^{-2}$ & 0.54 & 4.4 & 84 & 0.078 & 3.6 \\
\hline $6^{\mathrm{c}} \ldots \ldots \ldots \ldots \ldots \ldots$ & $p\left(q_{\mathrm{PB}}\right) \propto q_{\mathrm{PB}}^{1 / 2}$ & 0.92 & 7.6 & 101 & 0.079 & 3.7 \\
\hline 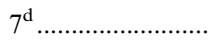 & $\left(M_{s}^{\mathrm{MT}}\right)_{\mathrm{th}}=20 M_{\odot}$ & 1.50 & 26.0 & 178 & 0.100 & 4.1 \\
\hline 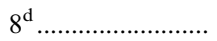 & $\left(M_{s}^{\mathrm{MT}}\right)_{\mathrm{th}}=30 M_{\odot}$ & 0.28 & 1.3 & 47 & 0.069 & 3.3 \\
\hline 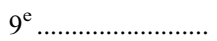 & $\sigma_{k}=100 \mathrm{~km} \mathrm{~s}^{-1}$ & 4.20 & 35.0 & 84 & 0.078 & 3.6 \\
\hline $10^{\mathrm{e}}$ & $\sigma_{k}=300 \mathrm{~km} \mathrm{~s}^{-1}$ & 0.15 & 1.3 & 85 & 0.079 & 3.6 \\
\hline $11^{\mathrm{f}}$ & $1.25 R_{c}$ & 0.14 & 2.1 & 157 & 0.084 & 4.9 \\
\hline $12^{\mathrm{f}}$ & $1.5 R_{c}$ & 0.02 & 0.4 & 280 & 0.093 & 5.8 \\
\hline $13^{\mathrm{g}}$ & $\left(\Delta M_{c, s}\right)_{\text {wind }}=0$ & 0.63 & 2.5 & 40 & 0.072 & 3.0 \\
\hline 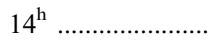 & $\Delta_{\mathrm{BH}}=0$ & 0.63 & 2.8 & 44 & 0.042 & 2.9 \\
\hline $15^{\mathrm{i}}$ & $\sigma_{k, \mathrm{BH}}=0 \mathrm{~km} \mathrm{~s}^{-1}$ & 0.63 & 4.8 & 76 & 0.058 & 3.6 \\
\hline $16^{\mathrm{i}} \ldots \ldots \ldots \ldots \ldots \ldots \ldots$ & $\sigma_{k, \mathrm{BH}}=400\left(M_{\mathrm{NS}} / M_{\mathrm{BH}}\right) \mathrm{km} \mathrm{s}^{-1}$ & 0.63 & 5.9 & 93 & 0.116 & 3.7 \\
\hline
\end{tabular}

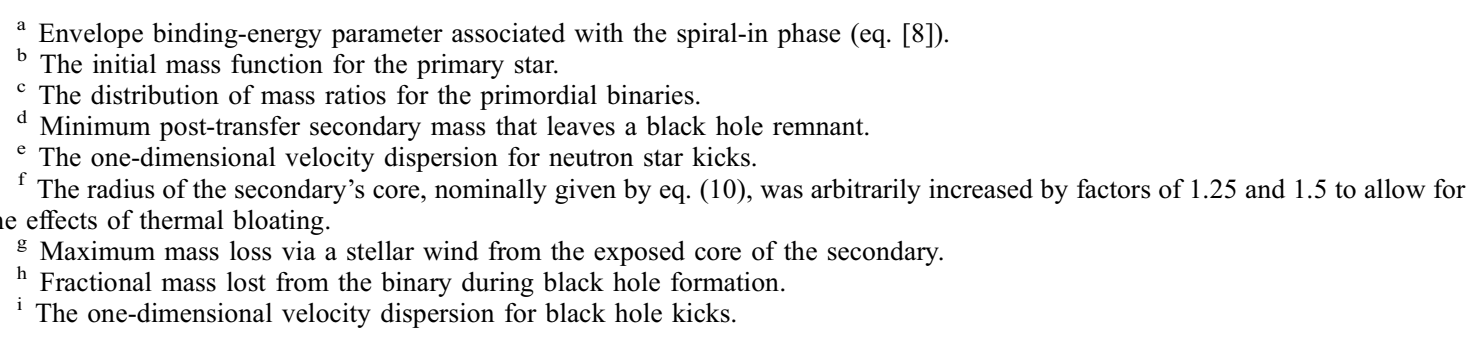

the neutron star can accept only $\sim 10^{-4} M_{\odot}$ of material. The short timescale, low accreted mass, and possibly low angular momentum of material accreted from a wind all suggest that pulsars in BHRPs may not be strongly recycled and could have systematically longer spin periods and higher surface magnetic fields than pulsars in DNSs (see Arzoumanian et al. 1999 for a related discussion and references).

\subsection{Collapse of the Secondary}

Uncertainties in black hole formation include the effects of winds from the massive progenitor and its exposed core in a binary system, stellar rotation, and the dynamics of core collapse (e.g., Fryer \& Kalogera 2001 and references therein). For the collapse, there are two possibilities. Either the core collapses promptly without being accompanied by a bright supernova (e.g., Gourgoulhon \& Haensel 1993), or a neutron star forms first and subsequently collapses to a black hole after accreting fallback material (Woosley \& Weaver 1995), ejecting a significant fraction of the envelope and resulting in a successful supernova. The two-stage scenario has been inferred for the black hole progenitor in the binary Nova Scorpii (GRO J1655-40; Podsiadlowski et al. 2002). Moreover, the high space velocity of Nova Sco requires that the black hole received a large natal kick of $\gtrsim 100 \mathrm{~km} \mathrm{~s}^{-1}$ (Brandt et al. 1995). It is not known whether most black holes receive significant kicks at birth (e.g., Fryer \& Kalogera 2001; Jonker \& Nelemans 2004). A plausible guess is that the kick momentum received by newborn black holes is similar to that received by neutron stars (see $\S 3.6$ ).

\section{FORMATION DETAILS}

Here we elaborate on the points in the last section. Each stage in the formation of a BHRP is described explicitly and quantitatively, although with a more transparent approach compared to many previous binary population synthesis studies. Our chosen assumptions and prescriptions for stellar evolution are fairly standard but are not unique. Further details and references may be found in PRP02.

\subsection{Primordial Binaries}

In our standard model, the mass of the primary is drawn from a power-law initial mass function, $p\left(M_{p}^{\mathrm{PB}}\right) \propto\left(M_{p}^{\mathrm{PB}}\right)^{-2.5}$, where $M_{p}^{\mathrm{PB}}$ is in the range of $8 M_{\odot}$ to $M_{\mathrm{th}}$, the mass threshold for black hole formation. Because of the uncertainty in $M_{\text {th }}$ and its possible dependence on binary evolution (see $\S 2.1$ ), we consider two scenarios, one in which $M_{\text {th }}=25 M_{\odot}$ (scenario I), and the other in which $M_{\mathrm{th}}=40 M_{\odot}$ (scenario II). The secondary mass $M_{\mathrm{SB}}^{\mathrm{PB}}$ is taken from a flat distribution in mass ratios, $q_{\mathrm{PB}}=$ $M_{s}^{\mathrm{PB}} / M_{p}^{\mathrm{PB}}<1$. Alternative choices for $p\left(M_{p}^{\mathrm{PB}}\right)$ and $p\left(q_{\mathrm{PB}}\right)$ are considered in $\S 5$ and Table 1 . Without much loss in generality, we assume that the orbit is circular, with a separation, $a_{\mathrm{PB}}$, drawn from a logarithmically flat distribution that extends from $\sim 10 R_{\odot}$ to a somewhat arbitrary maximum of $10^{6} R_{\odot}$.

\subsection{Stable Mass Transfer}

In isolation, the primary would grow to a maximum radius of $\gtrsim 1000 R_{\odot}$ before exploding as a supernova. Mass transfer from the primary occurs if it fills its Roche lobe of radius $R_{L}=$ $a_{\mathrm{PB}} r_{L}(q \mathrm{~PB})$, where

$$
r_{L}=\frac{0.49}{0.6+q_{\mathrm{PB}}^{2 / 3} \ln \left(1+q_{\mathrm{PB}}^{-1 / 3}\right)}
$$

(Eggleton 1983), with typical values of $r_{L}=0.5 \pm 0.1$. Mass transfer occurs over $\simeq 2$ decades in $a_{\mathrm{PB}}$, comprising $\simeq 40 \%$ of the binaries. 
Mass transfer may be stable or dynamically unstable depending on $q_{\mathrm{PB}}$ and the evolutionary state of the primary. If mass transfer is to be stable, then $q_{\mathrm{PB}}$ cannot be too small, and the primary should not be a deeply convective red supergiant (see PRP02 for a discussion, caveats, and references). In this regard, we assume that $q_{\mathrm{PB}}>0.5$, which is true for half of the binaries. By excluding very evolved primaries at the onset of mass transfer, we limit the stellar radius to less than $\simeq 200$ $1000 R_{\odot}$, where larger values correspond to higher masses. We also neglect systems where the primary transfers matter on the main sequence $\left(a_{\mathrm{PB}} \lesssim 30-40 R_{\odot}\right)$, which will not evolve into the objects of interest here. With these cuts, the net fraction of massive binaries that undergo stable mass transfer after the primary has left the main sequence is $f_{\mathrm{MT}} \sim 0.1$.

The primary is expected to lose its entire envelope during mass transfer, leaving its hydrogen-exhausted core of mass

$$
M_{c, p} \simeq 0.1\left(M_{p}^{\mathrm{PB}} / M_{\odot}\right)^{1.35} M_{\odot}
$$

(e.g., Hurley et al. 2000). Let $\beta$ be the fraction of transferred matter accreted by the secondary. The final secondary mass is then

$$
M_{s}^{\mathrm{MT}}=M_{s}^{\mathrm{PB}}+\beta\left(M_{p}^{\mathrm{PB}}-M_{c, p}\right) .
$$

We assume that the secondary is reset on the main sequence appropriate for its new mass, which is not justified for $q_{\mathrm{PB}}$ near 1.

Given $\beta$, it is straightforward to determine the distribution of $M_{s}^{\mathrm{MT}}$ given the distributions of $q_{\mathrm{PB}}$ and $M_{p}^{\mathrm{PB}}$. Figure 1 shows the resulting cumulative distribution of $M_{s}^{\mathrm{MT}}$ for $\beta=0.5$ and 1.0 in scenarios I and II. The fraction of systems in which the secondary collapses to a black hole and that remain potential BHRP progenitors can be estimated from Figure 1. If, in accord with the remarks in $\S 2.2$, we assume that all post-transfer secondaries of mass $>25 M_{\odot}$ leave black hole remnants, then for $\beta=0.5-1$, the fraction of systems in scenario $\mathrm{I}$ is $f_{\mathrm{BH}} \simeq$ $0.04-0.16$, while in scenario II the fraction is $f_{\mathrm{BH}} \simeq 0.12-0.23$. Setting the minimum secondary mass to $20 M_{\odot}$, we find that $f_{\mathrm{BH}}$ spans the range of $0.2-0.37$ and $0.13-0.3$ in scenarios I and II, respectively. Likewise, a minimum mass of $30 M_{\odot}$ gives fractions of $0.07-0.14$ and $0.006-0.14$ in the two scenarios.

The orbital separation following stable mass transfer, $a_{\mathrm{MT}}$, depends sensitively on $\beta$ and the specific orbital angular momentum taken by escaping matter. A plausible first approximation is that $a_{\mathrm{MT}} \sim a_{\mathrm{PB}}$. The resulting $\log a_{\mathrm{MT}}$ distribution is then roughly flat from $a_{\mathrm{MT}} \sim 0.1 \mathrm{AU}$ to several $\mathrm{AU}$, with a declining tail that extends to $\simeq 10 \mathrm{AU}$, which is correlated with higher post-transfer secondary masses. For separations $\gtrsim 10 \mathrm{AU}$, the primary cannot fill its Roche lobe during the mass transfer phase.

\subsection{Supernova of the Primary}

After mass transfer, $\lesssim 1$ Myr remains before the Type Ib/c supernova explosion of the primary's exposed core and formation of the neutron star, for which we assume a mass of $M_{\mathrm{NS}}=1.4 M_{\odot}$. Radial expansion of the naked core of the primary and wind mass loss do not affect our main results and are neglected. Associated with the core collapse and supernova is impulsive mass loss and a kick to the newborn neutron star. Measured proper motions of isolated radio pulsars suggest a median kick speed of 100-300 km s ${ }^{-1}$ (e.g., Hansen \& Phinney 1997; Arzoumanian et al. 2002). The functional form of the

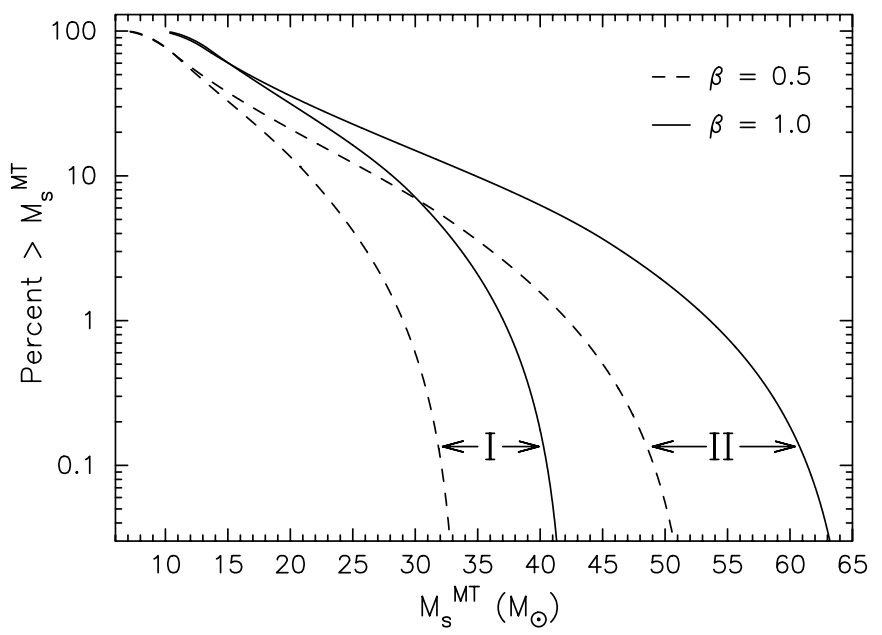

FIG. 1.-Cumulative distribution of secondary masses following stable mass transfer, where the primary initial mass function is $p\left(M_{p}^{\mathrm{PB}}\right) \propto\left(M_{p}^{\mathrm{PB}}\right)^{-2.5}$ for $8 M_{\odot}<M_{p}^{\mathrm{PB}}<M_{\mathrm{th}}$, and the secondary mass is drawn from a flat distribution in mass ratios such that $M_{s}^{\mathrm{PB}} / M_{p}^{\mathrm{PB}}>0.5$. The mass capture fraction is fixed at $\beta=$ 0.5 (dashed curves) or 1.0 (solid curves). Each pair of curves for a given $\beta$ corresponds to a different mass threshold for black hole formation from the primary: $M_{\mathrm{th}}=25 M_{\odot}$ (scenario I) or $M_{\mathrm{th}}=40 M_{\odot}$ (scenario II).

underlying kick distribution is weakly constrained. Moreover, there are observational and theoretical reasons to believe that the average kick magnitude may be much smaller for many neutron stars born in binary systems (Pfahl et al. 2002b; Podsiadlowski et al. 2004). We now quantify the dynamical effects of supernova mass loss and kicks on potential BHRP progenitors with $M_{s}^{\mathrm{MT}}>25 M_{\odot}$.

Since the primary's core mass is $\lesssim 8 M_{\odot}$, for BHRP progenitors the fractional binary mass lost in the supernova is

$$
\Delta_{\mathrm{NS}}=\frac{M_{c, p}-M_{\mathrm{NS}}}{M_{c, p}+M_{s}^{\mathrm{MT}}}<0.2,
$$

and so the dynamical effect of mass loss is relatively small. There is a significant probability for a binary to be unbound by the supernova if the neutron star kick speed, $v_{k}$, is comparable to or larger than the relative orbital speed of the binary components before the explosion:

$$
v_{b} \simeq 190 \mathrm{~km} \mathrm{~s}^{-1}\left(\frac{M_{b}^{\mathrm{MT}}}{40 M_{\odot}} \frac{1 \mathrm{AU}}{a_{\mathrm{MT}}}\right)^{1 / 2},
$$

where $M_{b}^{\mathrm{MT}}=M_{s}^{\mathrm{MT}}+M_{c, p}$. When $v_{k} / v_{b}=1$, the probability that the binary remains intact is roughly $50 \%$ if the kick directions are distributed isotropically. To be more quantitative, we must adopt a specific $v_{k}$ distribution.

A Maxwellian distribution, $p\left(v_{k}\right) \propto v_{k}^{2} \exp \left(-v_{k}^{2} / 2 \sigma_{k}^{2}\right)$, has the attractive features of being a reasonable characterization of the pulsar velocity data (for $\sigma_{k}=100-200 \mathrm{~km} \mathrm{~s}^{-1}$ ) and having only one free parameter. Figure 2, the result of a simple numerical integration, shows the survival probability versus $\sigma_{k} / v_{b}$ for an assumed fractional mass loss $\Delta_{\mathrm{NS}}=0.2$. In Figure 2 the probability asymptotically approaches $\simeq 0.64\left(\sigma_{k} / v_{b}\right)^{-3}$, a trend that is a direct consequence of the Maxwellian distribution. When $\sigma_{k}=100 \mathrm{~km} \mathrm{~s}^{-1}\left(200 \mathrm{~km} \mathrm{~s}^{-1}\right)$, the survival probability drops below $10 \%$ when $a_{\mathrm{MT}} \gtrsim 2.5 \mathrm{AU}(0.6 \mathrm{AU})$ for a reference binary mass of $40 M_{\odot}$.

More input is needed to estimate the net fraction of binaries that remain viable BHRP progenitors after the supernova. The 


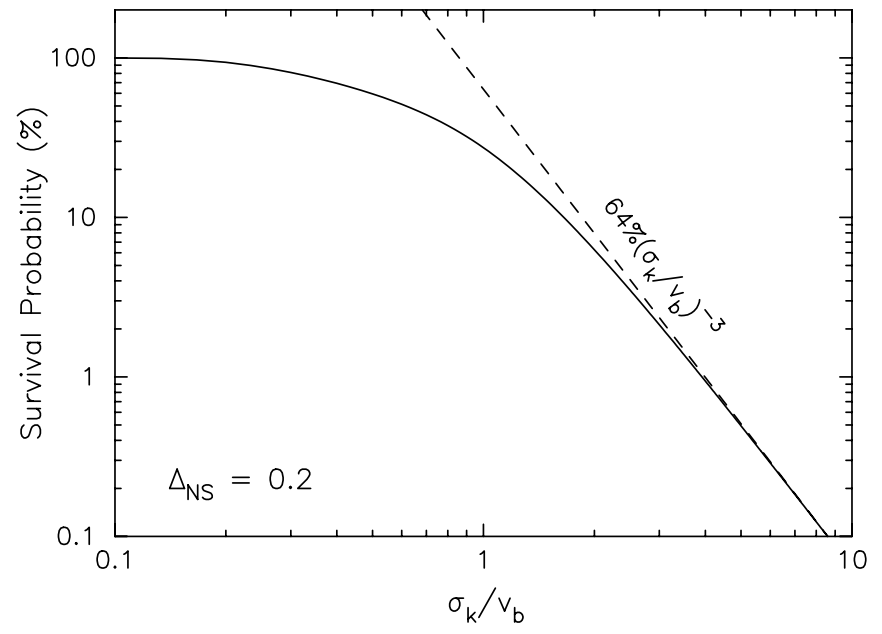

FIG. 2.-Supernova survival probability as a function of $\sigma_{k} / v_{b}$ for a Maxwellian kick-speed distribution, where $\sigma_{k}$ is the one-dimensional velocity dispersion. The probability is $<10 \%$ for $\sigma_{k} / v_{b} \gtrsim 1.7$ and $<1 \%$ for $\sigma_{k} / v_{b} \gtrsim 3.9$. For $\sigma_{k} / v_{b} \gtrsim 2$, the probability approaches the cubic trend $\simeq 64 \%\left(\sigma_{k} / v_{b}\right)^{-3}$ (dashed line).

semimajor axis and eccentricity after the supernova are given by

$$
\begin{gathered}
\left(\frac{a_{\mathrm{SN}}}{a_{\mathrm{MT}}}\right)^{-1}=1-\frac{\Delta_{\mathrm{NS}}+2 \xi w+w^{2}}{1-\Delta_{\mathrm{NS}}} \\
1-e_{\mathrm{SN}}^{2}=\left(\frac{a_{\mathrm{SN}}}{a_{\mathrm{MT}}}\right)^{-1} \frac{(1+\xi w)^{2}+\zeta^{2} w^{2}}{1-\Delta_{\mathrm{NS}}}
\end{gathered}
$$

(e.g., Brandt \& Podsiadlowski 1995), where $w=v_{k} / v_{b}$, and $v_{k} \xi$ and $v_{k} \zeta$ are the projections of the kick velocity onto, respectively, the directions of the pre-supernova orbital velocity and orbital angular momentum. After the supernova, the orbit circularizes to a separation of $a_{\mathrm{SN}, \mathrm{cir}}=a_{\mathrm{SN}}\left(1-e_{\mathrm{SN}}^{2}\right)$ - assuming conservation of orbital angular momentum-before the secondary fills its Roche lobe. At this stage, we desire the distribution of $a_{\mathrm{SN} \text {,cir }}$ for surviving binaries $\left(e_{\mathrm{SN}}<1\right)$. To that end, we have adopted the Maxwellian kick distribution, assumed for illustrative purposes that the $a_{\mathrm{MT}}$ distribution is logarithmically flat from 0.2 to $10 \mathrm{AU}$ and fixed the total binary mass at $40 M_{\odot}$. For reasons discussed in $\S 3.2$, this choice for the $a_{\mathrm{MT}}$ distribution may significantly overestimate the number of wide binaries. The elementary constraint $a_{\mathrm{SN}}\left(1-e_{\mathrm{SN}}\right)<a_{\mathrm{MT}}$ implies that $a_{\mathrm{SN} \text {.cir }}<2 a_{\mathrm{MT}}$ for $e_{\mathrm{SN}}<1$; thus, $a_{\mathrm{SN} \text {.cir }}<20 \mathrm{AU}$ in this example. Figure 3, the result of a simple Monte Carlo integration, shows the cumulative distribution of $a_{\mathrm{SN} . \text { cir }}$ for systems that remain bound. For $\sigma_{k}=50,100$, and $200 \mathrm{~km} \mathrm{~s}^{-1}$, the fraction of binaries with $a_{\mathrm{SN}, \mathrm{cir}}>10 \mathrm{AU}$ is $\simeq 2 \% \simeq 0.4 \%$, and $\simeq 0.07 \%$, respectively. Most systems with $a_{\mathrm{SN}, \text { cir }}<10 \mathrm{AU}$ $\left(\simeq 2000 R_{\odot}\right.$ ) will not survive the common-envelope phase, as discussed in $\S 2.2$. The cumulative probability scales approximately as $\sigma_{k}^{-2.5}$ for $a_{\mathrm{SN}, \mathrm{cir}}>5 \mathrm{AU}$. An increase in the total binary mass from 40 to $60 M_{\odot}$ increases the probabilities by at most a factor of 2 . In $\S 3.5$, we combine the results in Figure 3 with the severe constraints of the spiral-in phase in order to more precisely estimate the fraction of binaries that survive both the first supernova and the spiral-in.

Supernova mass loss and the neutron star kick impart some velocity to the binary center of mass. Considered independently, the contributions to the systemic speed from mass loss and the kick are, respectively, $v_{b} \Delta_{\mathrm{NS}}\left(1+M_{\mathrm{NS}} / M_{s}^{\mathrm{MT}}\right)^{-1}$ and

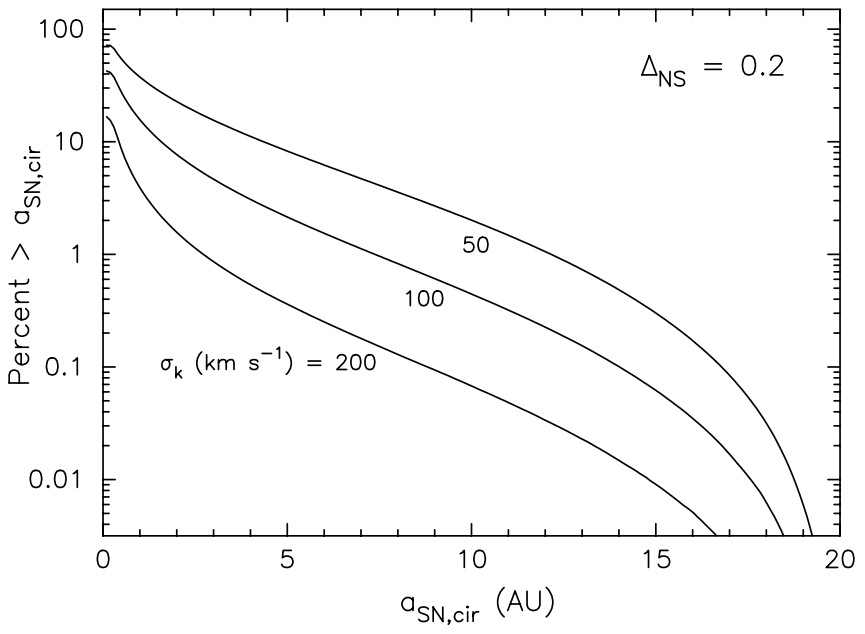

FIG. 3.-Cumulative distribution of circularized, post-supernova orbital separations, calculated under the assumptions that the presupernova binary mass is $40 M_{\odot}, 20 \%$ of the binary mass is lost in the explosion, and the distribution of presupernova orbital separations is logarithmically flat from 0.2 to $10 \mathrm{AU}$.

$v_{k}\left(1+M_{s}^{\mathrm{MT}} / M_{\mathrm{NS}}\right)^{-1}$ (e.g., Appendix B of PRP02). Most binaries that survive to ultimately form BHRPs will have $v_{b}$ and $v_{k}$ both less than $100 \mathrm{~km} \mathrm{~s}^{-1}$, since only wide systems can survive the common-envelope phase. Therefore, given that $\Delta_{\mathrm{NS}} \lesssim 0.2$ and $M_{\mathrm{NS}} / M_{s}^{\mathrm{MT}}<0.06$, the supernova of the primary's core imparts systemic speeds that are typically $<10 \mathrm{~km} \mathrm{~s}^{-1}$.

\subsection{Secondary Winds}

When the secondary's radius is $\gtrsim 1000 R_{\odot}$, it may lose most of its hydrogen envelope in a wind. A spherically symmetric wind that carries the specific orbital angular momentum of the star causes the orbit to expand at a rate of $\dot{a} / a=\dot{M}_{w} / M_{b}$, where $\dot{M}_{w}>0$ is the mass-loss rate and $M_{b}$ is the instantaneous binary mass. During this phase of wind mass loss, the Roche lobe radius of the secondary expands approximately in proportion to $M_{b}^{-0.85}$. If the stellar radius expands with a shallower mass dependence, which is quite possible for the masses of interest, then it will not be allowed to fill its Roche lobe at this stage (PRH03; see also Portegies Zwart et al. 1997 and Kalogera \& Webbink 1998 for related discussions). However, stellar winds from massive stars and the effects on binary evolution are very uncertain. The prescription for the angular momentum taken by the wind can be modified so as to limit or reverse the orbital expansion (see PRH03), making it possible for the secondary to fill its Roche lobe after its mass has been reduced significantly by the wind. This may lead to less extreme shrinkage during the common-envelope phase (see below), although it seems that the conditions that permit both Roche lobe overflow and significant mass loss may require fine tuning. This aspect of black hole binary formation deserves considerable attention.

\subsection{Common-Envelope Phase}

As the neutron star spirals in to the stellar envelope, frictional luminosity is generated at the expense of the orbital binding energy. If sufficient energy is available to disperse the envelope, the neutron star emerges in a tight orbit about the stellar core. A standard energetics argument (Webbink 1984) gives the orbital separation following the spiral-in:

$$
\frac{a_{\mathrm{CE}}}{a_{\mathrm{SN}, \mathrm{cir}}} \simeq \gamma \frac{r_{L}\left(q_{\mathrm{SN}}\right)}{2} \frac{M_{\mathrm{NS}} M_{c, s}}{M_{s}^{\mathrm{MT}} M_{e, s}},
$$


where $q_{\mathrm{SN}}=M_{\mathrm{NS}} / M_{s}^{\mathrm{MT}} \ll 1, M_{c, s}$, and $M_{e, s}$ are the secondary's core and envelope mass, and $\gamma$ is a combined parameter that quantifies the binding energy of the envelope and the efficiency with which orbital energy is used to expel the envelope. Over the full range of $M_{s}^{\mathrm{MT}}$, a good approximation to equation (8) is

$$
\frac{a_{\mathrm{CE}}}{a_{\mathrm{SN}, \mathrm{cir}}} \simeq 0.007 \gamma
$$

if stellar winds are neglected. Stellar winds prior to Roche lobe overflow reduce the envelope mass but not the core mass. A $50 \%$ reduction in the envelope mass increases $a_{\mathrm{CE}} / a_{\mathrm{SN}, \text { cir }}$ by a factor of $\simeq 3$, allowing a larger number of systems to avoid merger. However, as discussed in the last section, a star that experiences this degree of wind mass loss is not likely to fill its Roche lobe at all.

The structure parameter $\gamma$ varies as a given star evolves. Moreover, as the stellar mass is increased, the value of $\gamma$ at a particular evolutionary stage decreases systematically. Stars of mass $>25 M_{\odot}$ are expected to have $\gamma<0.1$ in the late stages of evolution (Dewi \& Tauris 2001; PRH03) and as low as $\sim 0.01$, giving contraction factors of $\gtrsim 1400$ from equation (8). The details of common-envelope evolution are poorly understood, especially the hydrodynamical aspects. Thus, there is considerable uncertainty in the appropriate $\gamma$, although it is difficult to see how the orbital contraction factor for the systems of interest could be much less than $1000(\gamma \simeq 0.14)$.

We now apply the results of $\S 3.3$ and illustrate how the fraction of systems that survive the spiral-in depends on $\gamma$. The core radius is estimated to be

$$
R_{c, s}=0.9\left(M_{c, s} / 10 M_{\odot}\right)^{0.6} R_{\odot}
$$

(J. Dewi \& O. Pols 2004, private communication). We assume a merger results if the core overflows its Roche lobe at the end of the spiral-in, i.e., if $a_{\mathrm{CE}}<R_{c, s} / r_{L}\left(q_{\mathrm{CE}}\right)$, where $q_{\mathrm{CE}}=M_{\mathrm{NS}} / M_{c, s}$. The merger condition then defines the allowed range in $a_{\mathrm{SN}, \text { cir }}$ :

$$
a_{\mathrm{SN}, \mathrm{cir}} \gtrsim 6.7 r_{L}^{-1}\left(q_{\mathrm{CE}}\right)\left(\frac{\gamma}{0.1}\right)^{-1}\left(\frac{R_{c, s}}{R_{\odot}}\right) \mathrm{AU} .
$$

We can now extract from Figure 3 the fraction, $f_{S}$, of systems that survive both the first supernova explosion and the common-envelope phase. Results are shown in Figure 4 for $\sigma_{k}=$ $200 \mathrm{~km} \mathrm{~s}^{-1}$ and secondary masses of $M_{s}^{\mathrm{MT}}=30$ and $50 M_{\odot}$. Smaller values of $f_{S}$ for the $50 M_{\odot}$ case are due to the larger radius of the exposed core. We have not taken into account the proper mass dependence of the supernova survival probability. However, this only partially makes up the difference between the two curves in Figure 4. Also shown in Figure 4 is a steep powerlaw approximation $\left(f_{S} \propto \gamma^{2.8}\right)$ to the survival fraction that works well for $\gamma>0.15$ but is clearly an overestimate when $\gamma \lesssim 0.1$. If this "fit" is combined with the $\sigma_{k}$ scaling from $\S 3.3$, we arrive at the simple approximate formula,

$$
f_{S} \sim 5 \times 10^{-4}\left(\frac{\sigma_{k}}{200 \mathrm{~km} \mathrm{~s}^{-1}}\right)^{-2.5}\left(\frac{\gamma}{0.1}\right)^{2.8} .
$$

The survival fraction that results from the above procedure assumes that the star can always grow large enough to fill its Roche lobe. In fact, the stars of interest probably grow no larger than $\simeq 2500 R_{\odot}$, which limits the maximum orbital separation to $\simeq 4000 R_{\odot}$ or $\simeq 19 \mathrm{AU}$. A smaller maximum radius only serves

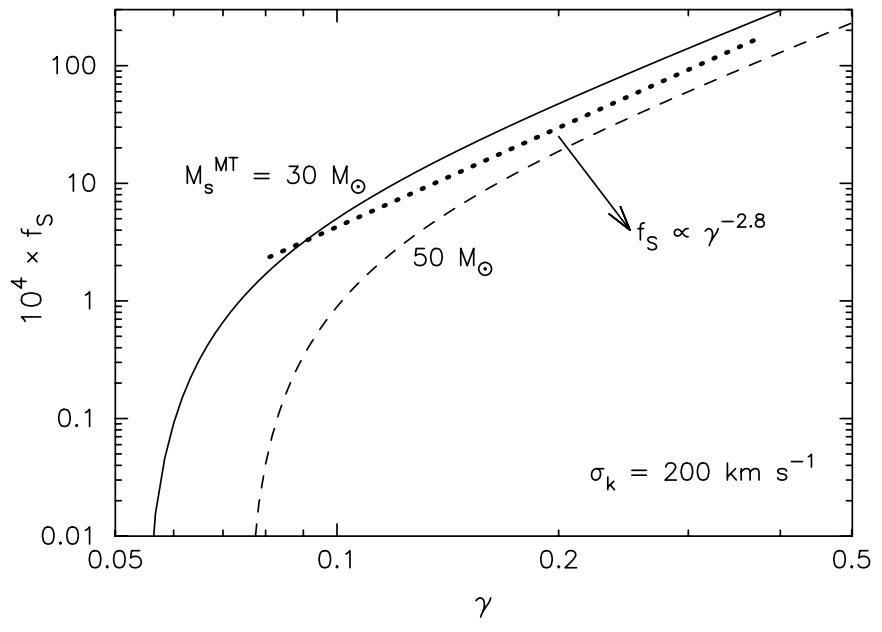

FIG. 4.-Fraction of systems that survive the both the first supernova and the spiral-in phase (see text for details), assuming $\sigma_{k}=200 \mathrm{~km} \mathrm{~s}^{-1}$. The two curves correspond to secondary masses of $M_{S}^{\mathrm{MT}}=30 M_{\odot}$ (solid curve) and $50 M_{\odot}$ (dashed curve). The thick dotted line shows a power-law approximation intermediate between the two curves for $\gamma \gtrsim 0.15$.

to decrease $f_{S}$, exacerbating the difficulty in forming BHRPs. If the orbit contracts by a factor of $>1000$ during the spiral-in, the resulting orbital separations are $\lesssim 4 R_{\odot}$, giving orbital periods of $\lesssim 7 \mathrm{hr}$ and relative orbital speeds of $\gtrsim 700 \mathrm{~km} \mathrm{~s}^{-1}$ for a total binary mass of $10 M_{\odot}$.

\subsection{Supernova of the Secondary and Final Products}

The severity of the orbital contraction in the commonenvelope phase implies that the orbit must initially be very wide and the secondary very evolved in order for the system to avoid merger. In most, if not all, cases the exposed core of the secondary will have already exhausted its central helium supply so that its remaining lifetime following the spiral-in is $\lesssim 10^{4} \mathrm{yr}$. At a mass-loss rate of $10^{-5}$ to $10^{-4} M_{\odot} \mathrm{yr}^{-1}$ (e.g., Chiosi \& Maeder 1986), stellar winds only have time to take away $\lesssim 1 M_{\odot}$, leading to a $\lesssim 10 \%$ widening of the orbit.

If the eventual core collapse is accompanied by a successful supernova explosion (see, however, $\S 2.4$ ), as much as half the mass of the secondary's core may be ejected, leading to a large orbital eccentricity. If there is no kick to the black hole, the postsupernova eccentricity is $e_{\mathrm{SN}}=\Delta_{\mathrm{BH}} /\left(1-\Delta_{\mathrm{BH}}\right)$, where $\Delta_{\mathrm{BH}}$ is the fractional mass loss from the binary (eqs. [6] and [7]). Black holes probably do receive kicks at birth, due to asymmetries in the collapse, explosion, or in the accretion of fallback material. However, even a black hole kick speed of $\sim 100 \mathrm{~km} \mathrm{~s}^{-1}$ - as inferred for Nova Sco (Brandt et al. 1995) - does not have a dramatic effect on the orbit, since the pre-supernova orbital speeds are $\gtrsim 700 \mathrm{~km} \mathrm{~s}^{-1}$. It is reasonable to suppose that, on average, black holes receive a kick momentum that is similar to neutron stars, leading to a typical kick speed that is perhaps $(100-300) \times\left(M_{\mathrm{NS}} / M_{\mathrm{BH}}\right) \mathrm{km} \mathrm{s}^{-1}$, with values of $\sim 10-80 \mathrm{~km} \mathrm{~s}^{-1}$ for $M_{\mathrm{BH}}=5-10 M_{\odot}$. If typical mass-loss and kick parameters are $\Delta_{\mathrm{BH}} \lesssim 0.3$ and $w \lesssim 0.2$, most newly formed BHRPs will have $e_{\mathrm{SN}} \lesssim 0.5$. Higher eccentricities are correlated with larger postsupernova semimajor axes, perhaps $\simeq 50 \%$ larger than the maximum separation following the spiral-in phase. In general, we expect that few systems are unbound at the time of black hole formation.

After the formation of the black hole, further orbital evolution is driven by the emission of gravitational radiation. The 
elapsed time before gravitational radiation causes the black hole and neutron star to merge is

$$
\tau_{m} \simeq 2.1 \operatorname{Myr} P_{h}^{8 / 3} M_{10}^{-2 / 3} \mu_{1}^{-1}\left(1-e^{2}\right)^{7 / 2},
$$

where $P_{h}$ and $e$ are the orbital period (in hours) and eccentricity, $M_{10}=\left(M_{\mathrm{NS}}+M_{\mathrm{BH}}\right) / 10 M_{\odot}$, and $\mu_{1}$ is the reduced mass in $M_{\odot}$. Equation (13) is our own fit (good to better than $20 \%$ for $e<0.9$ ) to the full integral expression for the merger time given in Peters (1964). BHRPs with relatively small eccentricities and periods of $5-10 \mathrm{hr}$ merge in $10^{8}-10^{9} \mathrm{yr}$.

In general, the mass loss and kick that coincide with the formation of the black hole make the largest contribution to the systemic velocity of a newly formed BHRP. Because the black hole dominates the mass of the binary, most of the kick velocity is transferred to the system; an average speed might be $20-30 \mathrm{~km} \mathrm{~s}^{-1}$ if the kick momenta are similar to neutron stars. If the average orbital separation following the spiral-in of the neutron star is 2-3 $R_{\odot}$, then the average pre-supernova orbital speed is $800-$ $1000 \mathrm{~km} \mathrm{~s}^{-1}$. Thus, the mean systemic speed due to impulsive mass loss in the second supernova may be $\simeq 20\left(\Delta_{\mathrm{BH}} / 0.1\right) \mathrm{km} \mathrm{s}^{-1}$, where $\Delta_{\mathrm{BH}}$ is a typical value in this context. A typical systemic speed of a few times $10 \mathrm{~km} \mathrm{~s}^{-1}$ results in a vertical scale height in the Galactic disk of a few times 100 pc. In contrast, it has been suggested that DNSs are born with mean systemic speeds of $\gtrsim 100 \mathrm{~km} \mathrm{~s}^{-1}$, acquired mainly after the second supernova, giving this population a scale height of $\gtrsim 1 \mathrm{kpc}$.

\section{BIRTHRATE ESTIMATE}

We take the total formation rate of massive binaries to be the core-collapse supernova rate, $\mathcal{R}_{\mathrm{SN}}\left(\sim 10^{-2} \mathrm{yr}^{-1}\right.$; Cappellaro et al. 1999), multiplied by the fraction, $f_{b} \simeq 0.5$, of stars in binaries. Thus, $f_{b} \mathcal{R}_{\mathrm{SN}}$ closely approximates the formation rate of the primordial binaries discussed in $\S 3.1$. Collected and summarized below are the efficiencies with which the initial ensemble of binaries passes through the various stages of evolution described above:

1. $f_{\mathrm{MT}}$. - The fraction of massive primordial binaries that undergo stable mass transfer after the primary has left the main sequence (see $\S \S 3.1$ and 3.2 for details). For a logarithmically flat distribution in initial orbital separations, $\simeq 10 \%$ of the massive binaries satisfy our criteria for stable mass transfer. Different assumptions that do not depart drastically from ours may lead to variations in $f_{\mathrm{MT}}$ by a factor of $2-3$.

2. $f_{\mathrm{BH}}$.- The fraction of post-transfer secondaries that ultimately leave black hole remnants. In addition to assumptions about the distributions of masses and orbital separations for the primordial binaries, this fraction depends on the mass threshold for black hole formation and on the amount of matter the secondary is able to accept from the primary. Figure 1 suggests that $f_{\mathrm{BH}} \simeq 0.04-0.2$.

3. $f_{S}$.- The fraction of systems that survive the supernova of the primary and avoid a merger following the spiral-in phase. Figure 4 shows the strong sensitivity of $f_{S}$ to $\gamma$ in the specific case of $\sigma_{k}=200 \mathrm{~km} \mathrm{~s}^{-1}$. An approximate scaling of $f_{S}$ with $\sigma_{k}$ and $\gamma$ is given in equation (12), which overestimates $f_{S}$ for $\gamma \lesssim 0.1$.

Combining these factors, we estimate the Galactic birthrate of BHRPs:

$$
\mathcal{B}=5 \times 10^{-8} \mathrm{yr}^{-1} \frac{\mathcal{R}}{0.01 \mathrm{yr}^{-1}} \frac{f_{b}}{0.5} \frac{f_{\mathrm{MT}}}{0.1} \frac{f_{\mathrm{BH}}}{0.1} \frac{f_{S}}{0.001}
$$

Both $f_{\mathrm{MT}}$ and $f_{\mathrm{BH}}$ could be made larger by a factor of 2 or 3 over their fiducial values in equation (14). It is then conceivable that, if both $f_{\mathrm{MT}}$ and $f_{\mathrm{BH}}$ are increased significantly, $\mathcal{B}$ could be as large as $\sim 10^{-6} \mathrm{yr}^{-1}$ even if $f_{S} \sim 10^{-3}$. However, it is important to note that $f_{S} \sim 10^{-3}$, which corresponds to $\gamma \gtrsim 0.1$, is quite optimistic in light of recent envelope binding energy calculations. We claim that a plausible upper limit to the rate is $\sim 10^{-7} \mathrm{yr}^{-1}$.

\section{GALACTIC POPULATION}

As the final step in our evolutionary study, we have combined the most important elements of $\S 3$ into a code designed to generate a population of newly formed BHRPs. Several free parameters are chosen via Monte Carlo methods from plausible distributions in such a way as to illustrate the possible range of outcomes. Fixed quantities in our simulation include the maximum primary mass of $30 M_{\odot}$ and the common-envelope parameter $\gamma=0.1$. After a BHRP is formed, we follow its further orbital evolution due to the emission of gravitational radiation. Our procedure is outlined below:

1. The initial primary mass is chosen from $p\left(M_{p}^{\mathrm{PB}}\right) \propto$ $\left(M_{p}^{\mathrm{PB}}\right)^{-2.5}$ over the range of $8-30 M_{\odot}$. The upper limit is intermediate between scenarios I and II discussed in $\S 3.1$.

2. The secondary mass, $M_{s}^{\mathrm{PB}}$, is drawn from a flat distribution in mass ratios. Systems with $M_{s}^{\mathrm{PB}} / M_{p}^{\mathrm{PB}}<0.5$ are dropped since the mass transfer from the primary is likely to be dynamically unstable (see $\S 3.2$ ).

3. The value of $\beta$ is chosen from the range $0.5-1$ with uniform probability. We then compute the secondary mass, $M_{s}^{\mathrm{MT}}$, after the phase of stable mass transfer from the primary. We adopt $25 M_{\odot}$ as the mass threshold for black hole formation from the secondary and cut systems with $M_{s}^{\mathrm{MT}}<25 M_{\odot}$.

4. Neglecting possible correlations between $M_{s}^{\mathrm{MT}}$ and the orbital parameters following the supernova, we assume $\sigma_{k}=$ $200 \mathrm{~km} \mathrm{~s}^{-1}$ and draw $a_{\mathrm{SN}, \text { cir }}$ from the cumulative distribution shown in Figure 3. As in Figure 3, we assume that the distribution vanishes at $a_{\mathrm{SN} \text {,cir }}=20 \mathrm{AU}$.

5. For a given secondary mass, we compute the mass and radius of the core (see eqs. [2] and [10]) that is exposed after the common-envelope phase. If the core overflows its Roche lobe following the spiral-in, the system is cut.

6. If a system survives the spiral-in, we assume that the secondary's core loses $0-2 M_{\odot}$ in a stellar wind, chosen with uniform probability. The orbit is widened accordingly.

7. We consider both impulsive mass loss and a kick to the black hole at the time of core collapse. The fractional mass loss is chosen uniformly from the range $0<\Delta_{\mathrm{BH}}<0.2$, and the kick is drawn from a Maxwellian distribution with $\sigma_{k, \mathrm{BH}}=$ $200\left(M_{\mathrm{NS}} / M_{\mathrm{BH}}\right) \mathrm{km} \mathrm{s}^{-1}$ so as to apply an average momentum kick that is similar to neutron stars.

8. We assume that BHRPs are formed at a constant rate and randomly choose a birth time, $t_{b}$, from the range 0 to $10 \mathrm{Gyr}$.

9. After the formation of the BHRP, further orbital evolution is driven by gravitational radiation, which we calculate using the equations of Peters (1964).

We followed $10^{9}$ binaries through the steps above. For each successful BHRP that is produced, the event is stored in a $500 \times 500$ array representing the distribution of initial orbital periods, $P_{b}$, and eccentricities, $e$. Figure 5 shows this distribution as a color image, where the colors were chosen according to the square root of the number of binaries formed in each element of the $P_{b}-e$ array. In going from magenta to yellow, the 


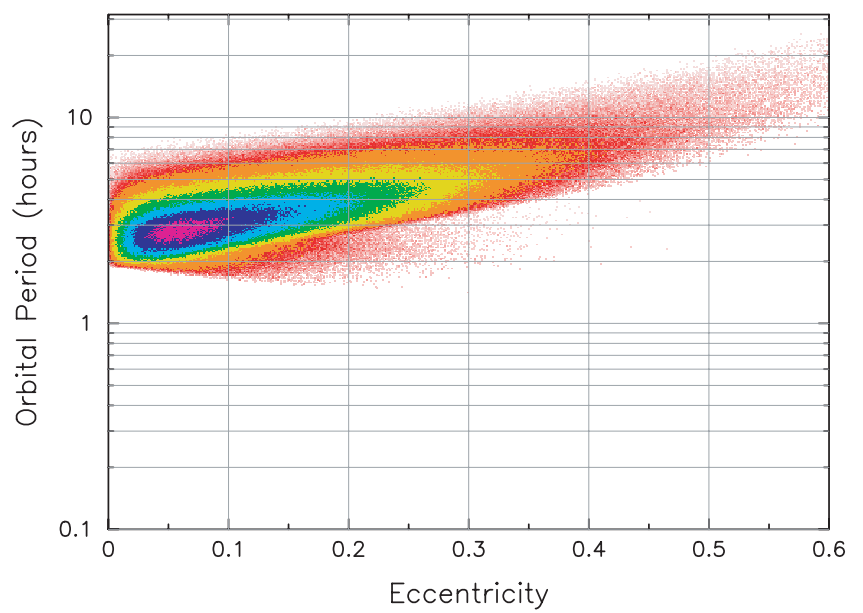

FIG. 5.-Distribution of orbital periods and eccentricities of newly formed BHRPs. The colors were chosen according to the square root of the number of systems that enter a given element of a $500 \times 500$ array. In going from magenta to yellow, the number drops by a factor of $\simeq 5$.

number per array element decreases by a factor of $\simeq 5$. Most of the newly formed BHRPs have $2 \mathrm{hr}<P_{b}<7 \mathrm{hr}$ and $e<0.3$. The effects of supernova mass loss and black hole kicks lead to a low-probability tail that extends to $P_{b} \simeq 25 \mathrm{hr}$ and $e \simeq 0.8$. Not displayed is the distribution of black hole masses, which ranges over $M_{\mathrm{BH}}=5-10 M_{\odot}$, with a mean of $\simeq 7 M_{\odot}$. Our Monte Carlo approach allows us to directly estimate the birthrate, since we are, in effect, explicitly calculating the survival fractions listed in $\S 4$.

Given the initial $P_{b}, e$, and $M_{\mathrm{BH}}$, the orbit is evolved forward in time using the equations of Peters (1964); $t_{e}$ is the evolutionary time since the birth of the BHRP. The evolution is terminated when the system merges for $t_{b}+t_{e}<10 \mathrm{Gyr}$, or when $t_{b}+t_{e}=10 \mathrm{Gyr}$ is reached. For each BHRP that traverses the $P_{b}$-e plane, we store the amount of time spent in each element of the $500 \times 500$ array; these times are accumulated for all the systems. The result is an array of total dwell times that represent the probability density of finding a system at the current epoch with a given $P_{b}$ and $e$. Figure 6 displays the expected currentepoch distribution of binary parameters.

Summing the dwell times over the entire array, dividing by the total number of BHRPs formed successfully from $10^{9}$ primordial binaries, and multiplying by the BHRP birthrate, we estimate the number of BHRPs that currently inhabit the Galaxy. In our standard model listed in the steps above, we find $\mathcal{B} \simeq 6 \times 10^{-8} \mathrm{yr}^{-1}$ and estimate that $\simeq 5$ BHRPs populate the Galaxy. This estimate can also be obtained upon examination of Figure 6, where it is evident that the average orbital period is $3-4 \mathrm{hr}$, corresponding to a short merger time of $\simeq(4-8) \times 10^{7} \mathrm{yr}$.

Table 1 gives an indication of how our results vary when we consider a range of plausible input assumptions that differ from those in our standard model. For each quantity or function that is varied, all else is left as in the standard model. As already demonstrated, the BHRP birthrate and total number in the Galaxy depend most strongly on the common-envelope parameter $\gamma$. In the very extreme case of $\gamma=0.3$, the birthrate and total number are $\sim 10^{-5} \mathrm{yr}^{-1}$ and $\simeq 4000$, respectively. For $\gamma=$ 0.15 , which is on the upper edge of plausibility, the total number is $\simeq 100$. Column (5), labeled $\left\langle\tau_{m}\right\rangle$, an average merger time, is defined to give the total number when multiplied by the birth-

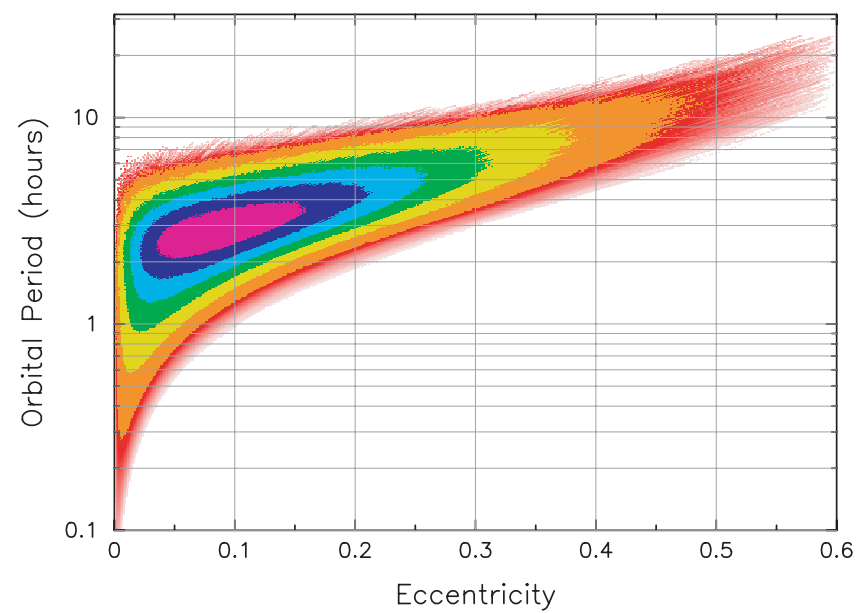

FIG. 6.-Distribution of orbital periods and eccentricities of BHRPs at the current epoch. The orbit of each binary has been evolved under the action of gravitational radiation losses. The colors are as in Fig. 5.

rate in column (3). In columns (6) and (7), $\langle e\rangle$ and $\left\langle P_{b}\right\rangle$ are traditional means of the distribution.

\section{PULSAR DETECTION AND TIMING}

Our results suggest that at most $\simeq 100$, but probably $\lesssim 10$, BHRPs currently populate the Galactic disk. We now consider what fraction of these systems are detectable in radio pulsar surveys. A plausible first guess is that, as a class, BHRPs are similar observationally to their DNS cousins. A number of papers (e.g., Kalogera et al. 2001 and references therein) indicate that $<1 \%$ of the total Galactic DNS population has been discovered among several extensive pulsar surveys conducted over the past $30 \mathrm{yr}$. This fraction incorporates the sensitivity and sky coverage of each survey, the expected spatial and luminosity distributions of DNSs, and the fraction $(\simeq 20 \%-30 \%)$ of pulsars beamed toward the Earth. Such a small detection efficiency is consistent with not yet having discovered a BHRP. If indeed there are $\simeq 100$ BHRPs in the Galaxy, then $\sim 1$ system may be within reach of ongoing surveys using the Parkes and Green Bank radio telescopes. However, we view this as being very optimistic, since $1 \%$ is probably a firm upper limit to the detection efficiency with current radio telescopes, and because we predict a substantially smaller number of BHRPs. Realistically, there are likely to be important differences between the BHRP and DNS populations that impact the BHRP detection likelihood, three of which we now discuss in turn.

The recycling histories of BHRPs and DNSs may be distinct. As discussed in $\S 2.2$, it is possible that pulsars in BHRPs have longer spin periods, $P_{s}$, and perhaps also larger surface magnetic fields, $B$. The spin-down luminosity scales as $B^{2} / P_{s}^{4}$. Systematically longer spin periods may be compensated for by larger magnetic fields, giving BHRPs luminosities similar to DNSs.

For reasons given in $\S 3.6$, the mean systemic speed (e.g., $\simeq 30 \mathrm{~km} \mathrm{~s}^{-1}$ ) of newly formed BHRPs may be smaller than for DNSs $\left(\gtrsim 100 \mathrm{~km} \mathrm{~s}^{-1}\right)$, resulting in a smaller scale height of BHRPs in the Galactic disk: $\lesssim 500$ pc for BHRPs, compared to $\gtrsim 1 \mathrm{kpc}$ for DNSs. Overall, we expect that the smaller scale height of BHRPs somewhat increases their discovery probability relative to DNSs but not by a large factor. It is also important to note that if BHRPs are more concentrated toward the Galactic midplane, pulse smearing due to dispersion in the interstellar 
medium, which decreases the sensitivity, is more significant than for DNSs. However, the modest relative reduction in sensitivity is partly canceled if pulsars in BHRPs have longer spin periods.

Perhaps the most robust difference between BHRPs and DNSs, in regard to their detectability, is that the pulsars in BHRPs should, on average, have larger orbital accelerations because of their more massive black hole companions and generally short orbital periods. Acceleration of the pulsar causes the measured pulse frequency to drift over the integration time of the observation, thus smearing the pulsar's signal in the Fourier power spectrum of the data and reducing its amplitude (e.g., Anderson 1993; Johnston \& Kulkarni 1991). For black hole masses in the range of 5-10 $M_{\odot}$, the acceleration of a pulsar in a BHRP is $\simeq 2$ times that in a DNS with the same orbital period and binary inclination, assuming that the orbits have small eccentricities. A search that explicitly probes for accelerated pulsars may be required in order to detect a BHRP. Such acceleration searches are computationally expensive, but are now done routinely.

Once a BHRP is discovered, the rewarding task of timing the pulsar begins. The initial Keplerian orbital parameters will be readily determined with high precision. Since BHRP orbits are probably very compact, the effects of relativistic gravity will be quite pronounced. Relativistic effects that have been measured in binary pulsars include orbital precession, the combined effect of the second-order Doppler shift and gravitational redshift (Einstein delay), the Shapiro propagation delay, orbital contraction due to gravity-wave emission, and the influence of geodetic precession on the measured pulse profile (Damour \& Taylor 1992; Weisberg \& Taylor 2002; Stairs et al. 2004). Each of these effects is a priori more significant in a BHRP than in a DNS, as are delays due to relativistic aberration and light bending (e.g., Wex \& Kopeikin 1999). The Keplerian parameters, coupled with the measurement of the Einstein or Shaprio delay, will yield the mass of the black hole, which is fundamentally important for studying models of black hole formation, in particular because the black holes in BHRPs have not accreted mass. A BHRP also offers the tantalizing possibility of measuring strong-field effects of relativistic gravity and providing stringent constraints on theories alternative to Einstein's general relativity. The high sensitivity of the planned Square
Kilometer Array may be required to tap into these more exotic problems (e.g., Kramer et al. 2004 and references therein) — and perhaps to provide the first BHRP detection.

\section{CONCLUSIONS}

We would like to underscore three main conclusions of our study.

1. There are major uncertainties in the formation of BHRPs, linked to important details in the life and death of stars more massive than $\simeq 20 M_{\odot}$, but mainly to a poor understanding of the common-envelope phase. Under quite optimistic circumstances, we expect that the Galactic BHRP birthrate is $\sim 10^{-7} \mathrm{yr}^{-1}$. The birthrate may be much lower, and possibly zero. The effects of dramatic orbital shrinkage during the common-envelope phase, prodigious stellar winds, and hypercritical accretion may preclude BHRP formation altogether (see $\S 2.2$ ).

2. Plausible, but possibly minimal, common-envelope shrinkage factors of $\simeq 1000$ lead to initial BHRP orbital periods of 2$10 \mathrm{hr}$ and an average merger time of $\sim 10^{8} \mathrm{yr}$.

3. The short merger times and low birthrate imply that there are most likely no more than $\sim 10$ BHRPs currently in the Galactic disk, making the prospects for their detection somewhat bleak. As a direct comparison, we estimate that there is probably no more than 1 BHRP in the Galaxy for every 100-1000 double neutron stars, of which only eight are currently known.

Our simulations suggest that most BHRPs at the current epoch have orbital periods of 1-6 hr. Due to supernova mass loss and possible black hole kicks, a typical eccentricity may be $\sim 0.1$. The acceleration of the pulsar in such a binary is large but does not greatly hinder its detection given current computational resources. If a BHRP is discovered, timing measurements would provide a wealth of new constraints on theories of gravitation.

We would like to thank J. Dewi, R. Di Stefano, A. Loeb, and S. Ransom for valuable discussions. E. P. was supported by NASA and the Chandra Postdoctoral Fellowship program through grant PF2-30024.

\section{REFERENCES}

Alpar, M. A., Cheng, A. F., Ruderman, M. A., \& Shaham, J. 1982, Nature, 300, 728 Anderson, S. B. 1993, Ph.D. thesis, Caltech

Arzoumanian, Z., Chernoff, D. F., \& Cordes, J. M. 2002, ApJ, 568, 289

Arzoumanian, Z., Cordes, J. M., \& Wasserman, I. 1999, ApJ, 520, 696

Belczynski, K., Kalogera, V., \& Bulik, T. 2002, ApJ, 572, 407

Bhattacharya, D., \& van den Heuvel, E. P. J. 1991, Phys. Rep., 203, 1

Brandt, N., \& Podsiadlowski, P. 1995, MNRAS, 274, 461

Brandt, W. N., Podsiadlowski, P., \& Sigurdsson, S. 1995, MNRAS, 277, L35

Brown, G. E. 1995, ApJ, 440, 270

Brown, G. E., Heger, A., Langer, N., Lee, C.-H., Wellstein, S., \& Bethe, H. 2001, NewA, 6, 457

Burgay, M., et al. 2003, Nature, 426, 531

Cappellaro, E., Evans, R., \& Turatto, M. 1999, A\&A, 351, 459

Chevalier, R. A. 1993, ApJ, 411, L33

. 1996, ApJ, 459, 322

Chiosi, C., \& Maeder, A. 1986, ARA\&A, 24, 329

Damour, T., \& Taylor, J. H. 1992, Phys. Rev. D, 45, 1840

Dewi, J. D. M., \& Pols, O. R. 2003, MNRAS, 344, 629

Dewi, J. D. M., \& Tauris, T. M. 2001, in ASP Conf. Ser. 229, Evolution of Binary and Multiple Star Systems, ed. Ph. Podsiadlowski et al. (San Francisco: ASP), 255

Eggleton, P. P. 1983, ApJ, 268, 368

Faulkner, A. J., et al. 2005, ApJ, 618, L119

Fryer, C. L., \& Kalogera, V. 2001, ApJ, 554, 548

Gourgoulhon, E., \& Haensel, P. 1993, A\&A, 271, 187

Hansen, B. M. S., \& Phinney, E. S. 1997, MNRAS, 291, 569

Hulse, R. A., \& Taylor, J. H. 1975, ApJ, 195, L51

Humphreys, R. M. 1984, in IAU Symp. 105, Observational Test of Stellar Evolution Theory, ed. A. Maeder \& A. Renzini (Dordrecht: Reidel), 279

Hurley, J. R., Pols, O. R., \& Tout, C. A. 2000, MNRAS, 315, 543

Ivanova, N., Belczynski, K., Kalogera, V., Rasio, F. A., \& Taam, R. E. 2003, ApJ, 592, 475

Johnston, H. M., \& Kulkarni, S. R. 1991, ApJ, 368, 504

Jonker, P. G., \& Nelemans, G. 2004, MNRAS, 354, 355

Joss, P. C., \& Rappaport, S. A. 1983, Nature, 304, 419

Kalogera, V., Narayan, R., Spergel, D. N., \& Taylor, J. H. 2001, ApJ, 556, 340

Kalogera, V., \& Webbink, R. F. 1998, ApJ, 493, 351

Kalogera, V., et al. 2004, ApJ, 601, L179

Kramer, M., Backer, D. C., Cordes, J. M., Lazio, T. J. W., Stappers, B. W., \& Johnston, S. 2004, NewA Rev., 48, 993

Langer, N., \& Maeder, A. 1995, A\&A, 295, 685

Lipunov, V. M., Postnov, K. A., Prokhorov, M. E., \& Osminkin, E. Y. 1994, ApJ, 423, L121

Lyne, A. G., et al. 2004, Science, 303, 1153

Narayan, R., Piran, T., \& Shemi, A. 1991, ApJ, 379, L17

Peters, P. C. 1964, Phys. Rev., 136, 1224

Pfahl, E., Rappaport, S., \& Podsiadlowski, P. 2002a, ApJ, 573, 283 (PRP02)

Pfahl, E., Rappaport, S., Podsiadlowski, P., \& Spruit, H. 2002b, ApJ, 574, 364

Podsiadlowski, Ph., Langer, N., Poelarends, A., Rappaport, S., Heger, A., \& Pfahl, E. 2004, ApJ, 612, 1044

Podsiadlowski, Ph., Nomoto, K., Maeda, K., Nakamura, T., Mazzali, P., \& Schmidt, B. 2002, ApJ, 567, 491 
Podsiadlowski, P., Rappaport, S., \& Han, Z. 2003, MNRAS, 341, 385 (PRH03)

Portegies Zwart, S. F., Verbunt, F., \& Ergma, E. 1997, A\&A, 321, 207

Sigurdsson, S. 2003, in ASP Conf. Ser. 302, Radio Pulsars, ed. M. Bailes, D. J. Nice, \& S. E. Thorsett (San Francisco: ASP), 391

Sipior, M. S., Portegies Zwart, S., \& Nelemans, G. 2004, MNRAS, 354, 49

Sipior, M. S., \& Sigurdsson, S. 2002, ApJ, 572, 962

Smarr, L. L., \& Blandford, R. 1976, ApJ, 207, 574

Stairs, I. H. 2004, Science, 304, 547

Stairs, I. H., Thorsett, S. E., \& Arzoumanian, Z. 2004, Phys. Rev. Lett., 93 , 141101
Tauris, T. M., \& van den Heuvel, E. P. J. 2003, to appear in Compact Stellar X-ray Sources, ed. W. H. G. Lewin \& M. van der Klis, astro-ph/0303456 Voss, R., \& Tauris, T. M. 2003, MNRAS, 342, 1169 Webbink, R. F. 1984, ApJ, 277, 355

Weisberg, J. M., \& Taylor, J. H. 2002, ApJ, 576, 942

Wellstein, S., \& Langer, N. 1999, A\&A, 350, 148

Wex, N., \& Kopeikin, S. M. 1999, ApJ, 514, 388

Woosely, S. E., \& Weaver, T. A. 1995, ApJS, 101, 181 